\title{
El desafío de incorporar las redes institucionales y comunitarias en la intervención con adolescentes infractores: una investigación- acción en tres regiones del sur de Chile*
}

\author{
The Challenge of Incorporating the Institutional and Community \\ Networks in the Intervention with Juvenile Offenders: An \\ Action Research in Three Southern Regions of Chile
}

Recibido: 1 de marzo de 2015 | Revisado: 1 junio de 2015 | Aceptado: 1 agosto de 2015

\author{
Alba Zambrano Constanzo ** \\ Universidad de La Frontera, Temuco, Chile \\ JAIME MUÑOZ VIDAL *** \\ Fundación Tierra de Esperanza, Chile \\ Claudio Andrade Gyllen ***** \\ Fundación Ciudad del Niño, CODENI, Chile
}

doi:10.11144/Javeriana.up14-4.diri

Para citar este artículo: Zambrano, A., Muñoz, J., \& Andrade, C. (2015). El desafío de incorporar las redes institucionales y comunitarias en la intervención con adolescentes infractores: una investigación-acción en tres regiones del sur de Chile. Universitas Psychologica, 14(4), 1371-1386. http://dx.doi.org/10.11144/Javeriana.up14-4.diri

* Artículo de Investigación. Este estudio se realizó en el marco del Proyecto FONDEF D08I1205 denominado "Estrategia ecosistémica especializada de intervención diferenciada para favorecer la integración psicosocial de adolescentes infractores de ley" financiado por CONICYT, en sociedad con el Servicio Nacional de Menores de Chile "SENAME", Fundación Tierra de Esperanza "FTDE" y Consejo de Defensa del Niño "CODENI", y es fruto de la colaboración de múltiples equipos de profesionales que intervienen con adolescentes infractores de ley en el sur de Chile.

** Alba Zambrano Constanzo: Directora Magister y Especialización en Psicología Comunitaria, Departamento de Psicología - Universidad de La Frontera

**** Jaime Muñoz Vidal: Psicólogo y Magíster en Psicología Comunitaria, Universidad de La Frontera, Chile. Coordinador Técnico Fundación Tierra de Esperanza, Chile.e-mail: jaime.munoz@tdesperanza.cl, jaime.munoz@ufrontera.cl

****** Claudio Andrade Gyllen: Sociólogo y Magíster en Psicología Comunitaria, Universidad de La Frontera, Chile. Investigador Departamento de Estudios, Fundación Ciudad del Niño, CODENI, Chile. e-mail: andradegyllen@gmail.com

\section{RES U MEN}

Se reportan los resultados obtenidos a partir de una investigación-acción con componentes participativos, orientada a fortalecer el funcionamiento de redes institucionales y comunitarias para la intervención con adolescentes infractores de ley. El artículo da cuenta de los principales resultados obtenidos a partir de este proceso, centrando su análisis en un conjunto de dimensiones que permiten tener una panorámica acerca de los avances y desafíos que presentan los programas involucrados en el estudio. Los resultados reportan las dificultades de los equipos para incluir de modo eficiente los recursos de las redes interinstitucionales dada la fragmentación de la política pública destinada a este grupo de adolescentes, así como las barreras para integrar la dimensión comunitaria en el trabajo con los jóvenes, producto del formato de intervención así como las modalidades de financiamiento de los programas. Igualmente, se evidencian mejoras en la comprensión e integración de estos componentes, por parte de los equipos de los programas durante el proceso, articulándose redes interinstitucionales con mejores grados de continuidad y complementariedad en su quehacer y una incorporación paulatina, aunque parcial, de la dimensión comunitaria en la intervención.

Palabras clave

adolescentes infractores de ley; programas de intervención; redes institucionales y comunitarias; investigación-acción

\section{A B S T R A C T}

The results of a participatory action research aiming the empowerment of institutional and community networks in the field of the intervention on youth offenders are presented. This paper focuses the analysis on several dimensions allowing us to have a broader perspective on the advances and challenges faced by intervention programmes on youth offenders. The results show the obstacles faced by these interventors in order to achieve efficiency in the use of resources within these networks. This is due to the fragmentation of public policies targeting these young offenders. It is also due to some barriers related to the inclusion of the community dimension within these programmes and also related to the mechanisms in place in order to provide funding. Some improvements in the understanding and integration of these dimensions by the interventors are also highlighted. Improvements in the continuity and complementarity of 
the work of these networks in order to increase the integration of the community level within the intervention are also found. Keywords juvenile offenders; intervention programs; institutional and community networks; action research

En lo que sigue de este apartado se realizará una breve revisión de antecedentes teóricos y empíricos que permiten, de modo general, ubicar la relevancia de considerar a las redes sociales en estrategias especializadas de atención a adolescentes infractores de ley. Caracterizando las particularidades del comportamiento trasgresor en población adolescente, se aportan antecedentes que argumentan el rol que juegan los ambientes sociales en las trayectorias de desadaptación social persistentes. Luego, se señalan los desafíos específicos que deben enfrentar los equipos especializados para mejorar la continuidad y complementariedad de la intervención dirigida a los adolescentes que presentan comportamiento trasgresor persistente. Así también, se destaca la necesidad de integrar estratégicamente recursos para favorecer la inclusión sociocomunitaria de estos adolescentes, lo cual supone un esfuerzo sostenido y deliberado de articulación de redes focales, comunitarias e institucionales.

\section{El comportamiento infractor de ley en población adolescente}

Las investigaciones de las últimas décadas acerca de la delincuencia juvenil han llegado a un cierto consenso respecto al menos de dos cuestiones fundamentales, la primera refiere la necesidad de comprender la delincuencia en esta etapa del desarrollo como una expresión de las particularidades evolutivas. Así, probar límites, incurrir en comportamientos de riesgos sin valorar apropiadamente las consecuencias, experimentar emociones, además de la influencia del grupo de pares, entre otros aspectos, estarían a la base de este comportamiento transitorio de trasgresión de normas. Una segunda cuestión es la necesidad de establecer una distinción entre esta forma transitoria de transgresión, del comportamiento delictivo persistente en los adolescentes que cometen delitos. El segundo tipo delictivo se asocia a la reiteración y la precocidad en conductas trasgresoras (Dionne \& Altamirano, 2012; Dionne \& Zambrano, 2009; Fréchette \& LeBlanc, 1998; Gendreau, Goggin, Cullen, \& Andrews, 2000; Hoge \& Andrews, 1994). En este caso, existiría a la base un conjunto de factores de riesgo que ha favorecido esta condición, y que por tanto implica una serie de vacíos educativos y necesidades del desarrollo que deben ser abordadas de modo especializado.

Por tanto, abordar la delincuencia en la etapa juvenil requiere de una respuesta diferenciada y especializada (Dionne \& Altamirano, 2012; Dionne \& Zambrano, 2009). En la delincuencia persistente se necesita abordar sistémicamente las necesidades del desarrollo así como los riesgos criminógenos subyacentes a la conducta infractora, lo que supone contar con condiciones en el contexto de vida del adolescente y en los dispositivos institucionales especializados para atender apropiadamente a su complejidad. Más aún, si se considera que los adolescentes que presentan delincuencia persistente son un grupo heterogéneo, que presenta diferentes trayectorias delictivas (Pérez-Luco et al., 2014) que requieren de una intervención específica para cada una de ellas.

Sin embargo, en la actualidad, la política pública en muchos países de Latinoamérica presenta reduccionismos que "recortan" el accionar estatal en líneas de acción paralelas, descoordinadas y a veces contradictorias (Repetto, 2010). En el caso chileno, esta constatación es confirmada en el estudio de caso de diferentes políticas públicas (Delpiano, 2011; Vega, 2012), observándose una escasa presencia de trabajo concertado en los contextos de vida de los adolescentes que presentan problemas. En particular en la experiencia con adolescentes infractores de ley, se aprecian importantes discontinuidades, incoherencia sistémica, fragmentación y baja pertinencia de la oferta pública en la atención de este grupo (Zambrano, Muñoz, \& Andrade, 2014).

Es de relevancia, entonces, favorecer mecanismos que contribuyan a mejorar la articulación y coherencia de las respuestas institucionales para contar con una plataforma apropiada para la intervención especializada y diferenciada que requieren 
los adolescentes infractores de ley, particularmente aquellos que viven procesos más complejos de desadaptación y exclusión social.

\section{El rol del contexto relacional y de los recursos sociocomunitarios en los procesos de desadaptación social}

Una perspectiva que destaca el rol de lo relacional en los procesos de desadaptación social, especialmente la delincuencia, es la planteada por LeBlanc, Swischer, Vitaro y Tremblay (2007), quienes proponen que la acumulación de experiencias relacionales negativas en el ambiente social potencia la presencia de factores de riesgo de desadaptación en niños y adolescentes que muestran algunas dificultades iniciales de comportamiento.

En el caso específico de los adolescentes que presentan comportamiento delictivo, se evidencia que el proceso de acceso a apoyo prosocial se ve muchas veces disminuido a partir de la vinculación con figuras contraculturales (Crespi \& Mikulic, 2009; Méndez \& Barra, 2008; Sandoval, 2007). Ello se puede asociar a un progresivo empobrecimiento vincular denominado por Bonet i Martí (2006) como vulnerabilidad relacional. Este concepto permite comprender que adolescentes que viven en entornos con redes sociales que no tienen acceso a recursos para resolver necesidades del desarrollo quedan aislados de oportunidades, reforzándose condiciones de marginalidad. Este concepto puede también aplicarse a la experiencia de adolescentes que comparten su vida con otros adolescentes en ambientes criminógenos y que desarrollan recursos relacionales que los alejan de entornos convencionales.

Considerando esta complejidad y el carácter ecosistémico de la conducta transgresora en los adolescentes, Canales et al. (2005) plantean la necesidad de abordar su evolución desde un enfoque integrado con carácter psicosocial que incorpore todas las áreas de la vida de los sujetos. Una forma de operacionalizar los niveles que requieren ser abordados, consiste en estructurar el entorno social de vida de los individuos, delimitando niveles de análisis que van desde el área más próxima a la persona hasta llegar al mundo institucional. Desde esta perspectiva, se pueden distinguir al menos tres niveles claramente diferenciados en el contexto de vida.

En primer lugar, las redes focales, que en el caso de los adolescentes quedan constituidas principalmente por el grupo de pares (Claes et al., 2005; Holt, Bossler, \& May, 2011; Mears \& Field, 2002; Moreira, Sánchez, \& Mirón, 2010; Rezende \& Estevão, 2012; Rodríguez, 2011; Rodríguez \& Mirón, 2008).

En segundo lugar, la comunidad de pertenencia o el contexto sociocomunitario más próximo a la vida del adolescente, constituido principalmente por los barrios en los que estos viven. En ellos, pueden existir variables que actúan como protección o riesgo para la realización de conductas desadaptativas. Entre estas últimas destacan: la existencia de grupos de pares desadaptativos, la desorganización y fragilización comunitaria y cultural, la débil aplicación de controles comunitarios, la normalización del consumo de drogas y la percepción de fácil acceso a la adquisición de armas de fuego, niveles disminuidos de participación, apoyo y cohesión social, así como una identidad barrial negativa (Hein, 2002; LeBlanc, 2006; Serrano et al., 2009; Valdenegro, 2005; Zambrano, Muñoz, \& González, 2012).

En tercer lugar, y por último, la dimensión institucional. En ella, pueden estar presentes recursos para la inclusión social o, en contraparte, dinámicas que eventualmente favorecen la victimización o cronificación de dificultades de los jóvenes, especialmente si ellos han presentado problemas desde temprano en su desarrollo y han vivido en contextos que no han atendido oportunamente sus necesidades (Martínez, 2010; Veillet \& Laporte, 2010).

Cada nivel posee una lógica diferente de análisis, puesto que una red focal puede ser vista en función de un caso específico, pero un barrio deberá ser analizado desmarcándose del caso, al igual que las relaciones institucionales que van incluso más allá del análisis de un barrio específico, y bien podrían ser observadas en función de una ciudad, una provincia o una región.

Las redes focales son definidas por Martínez (2006, p. 76) como: "sistemas de conversaciónacción que se estructuran en torno a un sujeto foco, 
que puede ser una persona, una pareja, una familia, un pequeño grupo, constituyendo el ecomapa de dicho sujeto, es decir, el mapa de las relaciones sociales de su entorno próximo", por las cuales, según Gracia y Herrero (2006), circula apoyo social. Por su parte, la comunidad corresponde a un grupo más amplio que las redes focales donde se encuentra, dado su carácter dinámico, en constante transformación y evolución, generando un sentido de pertenencia e identidad social para sus integrantes, a partir de las interacciones de los actores que la componen (Montero, 2005).

Las relaciones interinstitucionales, se entienden como: "sistemas de conversación acción de carácter amplio y flexible de articulaciones multidimensionales entre organizaciones, instituciones, asociaciones, grupos y actores individuales" (Martínez, 2006, p. 78). En este nivel, las relaciones necesitan orientarse con base en una lógica horizontal, de modo de favorecer la diversidad y el diálogo entre los diversos actores (Fleury, 2002). En este sentido, las características de las instituciones, que se ponen de manifiesto en sus formas de vinculación con otras instituciones, con la comunidad y con las personas en general, pueden impactar en las potencialidades de estas últimas de participar de forma activa en la configuración de estrategias para resolver los problemas que abordan.

Entonces, se puede plantear que dependiendo de cómo se organicen y funcionen los diversos niveles del entorno relacional de los adolescentes infractores de ley, pueden aumentar o disminuir las probabilidades de que mantengan o superen el comportamiento delictivo o de riesgo. De este modo, un programa que intervenga con adolescentes infractores de ley, y que esté orientado a su inclusión social y comunitaria, debe identificar, caracterizar y gestionar de forma estratégica los recursos presentes en los tres niveles ya mencionados. En síntesis, desde la perspectiva ecosistémica, para mejorar los entornos relacionales y emplear los recursos disponibles (visibles, latentes o potenciales) en una intervención con adolescentes infractores de ley, se requiere actuar en estos diferentes niveles.

Al revisar la evidencia aportada a nivel internacional por programas que buscan prevenir la delincuencia y reincidencia delictiva, se aprecia el valor asignado a estrategias que incluyen la dimensión comunitaria y la vinculación positiva con redes institucionales. El programa "Todos somos Juárez" (Rendón, 2013) destaca la necesidad de intervenir en las condiciones contextuales de los entornos que concentran factores de criminalidad, a partir de estrategias participativas, con un fuerte componente de inclusión social de los adolescentes que presentan dificultades. En esta misma línea, el Programa Nacional Escolhas (Vieira, 2013) aporta evidencia a partir de lo realizado en distintos barrios de alto riesgo y vulnerabilidad social, en Portugal. Este programa, con más de una década de implementación, favorece la acción territorial coordinada de distintas instituciones y organizaciones que combinan estrategias de promoción y prevención focalizada en los grupos de alto riesgo.

El Programa de Prevención y Mediación Comunitaria de la Dirección General de Ejecución Penal en la Comunidad y Justicia Juvenil del Departamento de Justicia de la Generalitat de Catalunya es otra experiencia que enfatiza el valor de la articulación de recursos locales para favorecer políticas sociales orientadas a desarrollar acciones preventivas normalizadoras e integradoras de los jóvenes en conflicto social. Su finalidad es atender y apoyar a los gobiernos locales en el abordaje de las situaciones conflictivas en las que están implicados los niños y jóvenes del municipio, para lo cual se emplea metodología participativa, implicando a diferentes áreas y servicios que los atienden, comprometiendo a la comunidad para afrontar la situación de esta población y adaptando las respuestas a la realidad de cada localidad (Gimeno \& Nogueras, 2013).

En un estudio reciente que analiza los discursos de expertos en torno a los componentes y criterios fundamentales para estrategias de prevención comunitaria de desadaptación social y comportamiento de riesgo adolescente (Zambrano, Betancourt, Jerez, Ortiz, \& Soto, 2014), se consigna que las redes sociales e institucionales deben articularse con el fin de brindar apoyo a las familias para que estas puedan cumplir su rol protector, atendiendo a las necesidades de los adolescentes. El sentido de la 
intervención debiera orientarse a generar un anclaje de la comunidad con las instituciones que facilite la autonomía y brinde oportunidades de desarrollo para los jóvenes.

Dionne y Altamirano (2012), con base en la experiencia de seis decenios de la provincia de Quebec (Canadá) y la literatura científica, destacan como condición importante para desarrollar intervenciones de mayor eficacia, que los diferentes actores y las instancias responsables de la aplicación de sentencias y de la reeducación sean competentes y trabajen concertadamente. La colaboración debe darse en los diferentes niveles involucrados, ello es, entre profesionales y familiares del joven; entre organismos del sistema social y organismos del sistema de justicia; entre los profesionales de los programas de justicia juvenil y los miembros de la comunidad y entre los investigadores y quienes intervienen en el terreno.

En contraste, estudios realizados en Chile señalan que existiría poca o nula coordinación en la atención que se brinda a la población infantojuvenil que se encuentra en dificultades (Contreras, Rojas, $\&$ Contreras, 2015). Para la población adolescente infractora, esta realidad sería particularmente grave, pues al menos parte de los adolescentes mostrarían un conjunto de dificultades psicosociales que requieren de un trabajo concertado multinivel que garantice integralidad (Zambrano, Muñoz et al., 2014).

Es en este contexto que este estudio propone caracterizar la incorporación de las redes interinstitucionales y comunitarias en las intervenciones con adolescentes infractores de ley, por parte de los equipos de programas destinados a esta población en tres regiones del sur de Chile: La Araucanía, Los Ríos y Los Lagos. Se caracteriza el proceso considerando una medición en dos tiempos; así mismo, se analiza de modo global el proceso de las redes interinstitucionales coordinadas a partir de la investigación-acción, detectando los avances de los equipos para incluir de modo deliberado y coordinado el componente sociocomunitario en las intervenciones con adolescentes infractores de ley, como también las condiciones para sostener un proceso de articulación de mayor alcance.
Se operó bajo el supuesto de que a partir de un proceso participativo de investigación-acción se mejorarían los niveles de coordinación entre programas que aplican sanciones, facilitando condiciones para perfeccionar la inclusión a nivel local, comunal y regional de otras entidades que operan o gestionan recursos requeridos por los adolescentes para optimizar su actual condición. Así, se supone también que un proceso de esta naturaleza favorecerá la apertura a la formación e integración de herramientas teóricas y prácticas que favorezcan la incorporación de las redes más próximas a los adolescentes en los procesos de intervención contextual que debieran realizar los equipos para facilitar procesos de su inclusión prosocial.

\section{Metodología}

El enfoque metodológico empleado es la investigación-acción con componentes participativos, en un proceso desarrollado entre los años 2011 y 2013. Desde este enfoque, en la experiencia se procuró integrar a) investigación, en tanto que se trata de un componente reflexivo, sistemático, controlado y crítico, en el cual se estudia la realidad con una finalidad práctica; b) acción, dado que realizar el estudio es un tipo de intervención orientada a generar cambios intencionados y c) participación, que involucra tanto al investigador como a los destinatarios, quienes no son considerados como objetos de investigación, sino como sujetos activos en la realización de la misma. De modo específico, el diseño consideró evaluación en dos tiempos con etapas recursivas, que denominaremos situación inicial (T1) y segundo momento (T2). Cada uno de los tiempos se ordena en un ciclo compuesto por cuatro etapas: 1. Diagnóstico-Línea Base; 2. DevoluciónProblematización; 3. Definición de orientaciones, acciones, compromisos para mejorar la gestión de redes y resultados; 4. Evaluación e identificación de nuevas líneas de acción (Figura 1).

En este artículo, se reportan los principales cambios obtenidos a partir del proceso, utilizando como fuentes de datos los resultados obtenidos en la etapa 1 , de diagnóstico y línea base, los cuales 


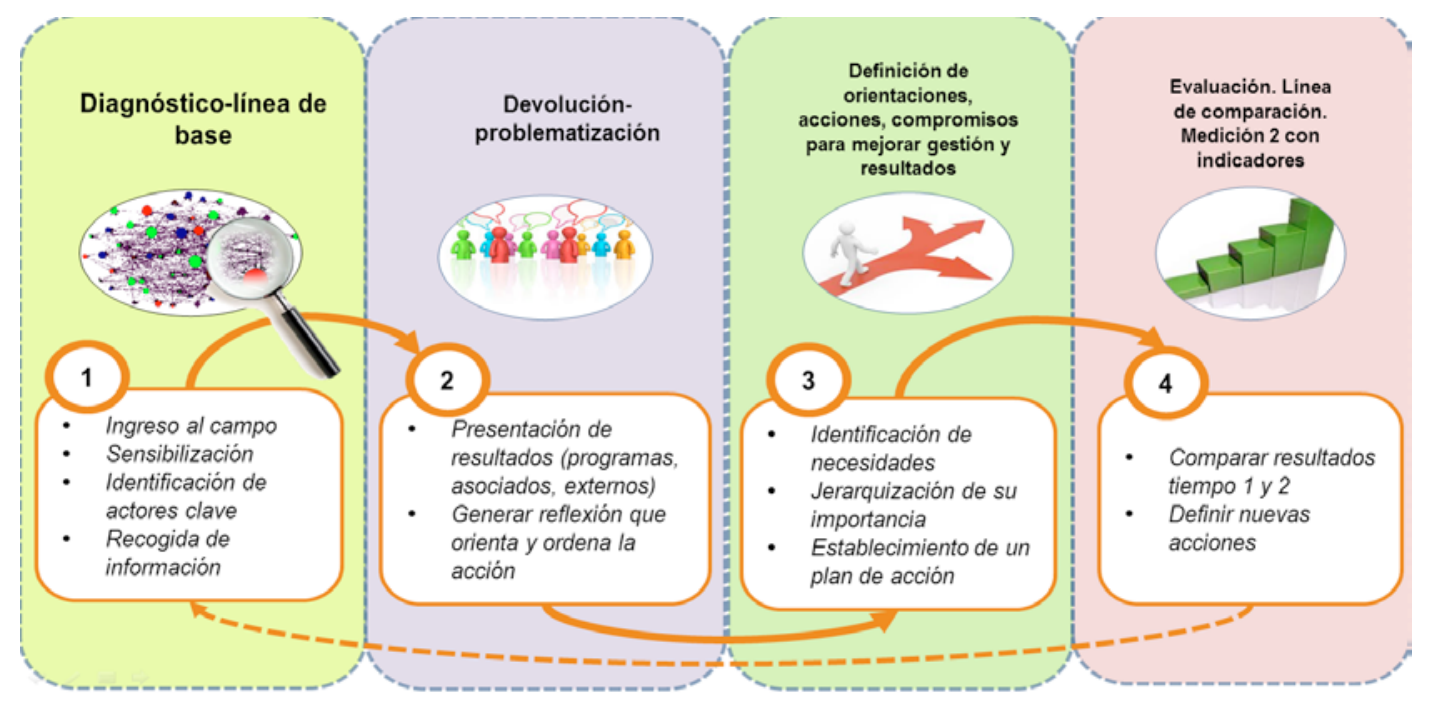

Figura 1. Las etapas del proceso de investigación-intervención con redes institucionales.

Fuente: elaboración propia

se comparan con datos recabados en la etapa 4, denominada evaluación, donde se constituye una línea de comparación con unos ciertos indicadores que permiten dar cuenta de los avances obtenidos hasta ese momento.

Los participantes fueron los equipos que aplican sanciones penales tanto en medio libre de diferentes entidades colaboradoras del Servicio Nacional de Menores (en adelante SENAME) en las tres regiones: Fundación Tierra de Esperanza, Corporación del Desarrollo del Niño, Proyecta, Fundación CIEM Villarrica, así como en los centros de administración directa del SENAME, responsables de los programas en medio cerrado y semicerrado. También participaron las Unidades de Justicia Juvenil del SENAME en las tres regiones involucradas en el estudio. Se contó, en algunas regiones, con la participación de los equipos ejecutores del Servicio Nacional para la Prevención y Rehabilitación del Consumo de Drogas y Alcohol (SENDA) y direcciones regionales y provinciales de salud, educación y capacitación. El número de personas durante el proceso fluctuó según se realizaran actividades por equipo, actividades entre los diferentes programas o encuentros más amplios en que se incluyeron profesionales de las redes regionales (en las tres regiones participó un número total estimado de 150 personas).
Las técnicas de producción de datos combinaron entrevistas focalizadas (aplicadas a encargados de programas y servicios y a integrantes de los equipos de los programas que trabajan directamente con adolescentes infractores de ley, para un total 60 entrevistas), observación participante, talleres grupales de análisis, herramientas de análisis de redes (sociogramas, cuestionarios generadores de nombres, aplicados en los equipos de los programas que aplican sanciones en medio libre, cerrado y semicerrado). Se combinó todo esto con herramientas participativas de análisis temático y de discusión de resultados en el proceso, así como estrategias participativas de planificación y evaluación de avances con los equipos de los programas y la mesa de trabajo de cada región, que tuvieron una frecuencia trimestral durante la duración de la investigación. En materia de análisis de datos, se combinaron también en función de la naturaleza de los datos, análisis de contenido (en la lógica de análisis en progreso) y análisis estructural de redes. La participación fue acordada con las y los diferentes profesionales, las sistematizaciones parciales fueron compartidas y contrachequeadas, empleándose como material para ir alimentando el proceso de reflexión-acción. El modelo derivado de la experiencia fue sistematizado en un manual de redes y puesto a disposición de los diferentes equipos y entidades (Zambrano et al., 2014). 


\section{Resultados}

Los resultados obtenidos en el estudio serán presentados de un modo global, y dado el volumen de la información obtenida se acudirá a ejemplos específicos para ilustrar algunos aspectos que conviene destacar.

De forma panorámica, se puede plantear que ninguna de las tres regiones presentaba un trabajo articulado de todos los programas que aplican sanciones a adolescentes que permitiera definir una estrategia mancumunada de trabajo con otras instituciones cuyos recursos son requeridos para la intervención con los adolescentes. Los niveles de coordinación y complementariedad fluctuaba entre regiones, apreciándose mayor articulación en la región de Los Lagos, en tanto en la región de la Araucanía existía una coordinación de tipo formal propiciada desde la Unidad de Justicia Juvenil del SENAME, sin mayor avance en la colaboración entre programas. En el caso de la región de Los Ríos, se aprecia al partir una baja especialización de los equipos de justicia juvenil, requiriendose fortalecer el trabajo al interior de los programas, previo a iniciar un trabajo de mayor alcance en lo que a la concertación de redes se refiere. En las tres regiones, existían esfuerzos radicados en cada programa para vincular redes institucionales que resolvieran necesidades específicas de intervención.

En un balance de conjunto, se aprecian cambios en el T2 respecto de T1 en distintos ámbitos, tales como: la valoración de lo relacional en los equipos de intervención, modificaciones en la estructura de las redes con las que cuentan los programas, transformaciones en la dinámica o nuevas formas de funcionamiento de las redes, avances en la incorporación del ámbito territorial a la intervención y la visión diferenciada de la intervención en redes, lo que implica la generación de contextos relacionales asociados a las características particulares de los casos atendidos y sus necesidades de intervención diferenciada. Por cierto, se aprecian diferencias en los grados de avance en las regiones, localidades y programas involucrados.

En T1 se aprecia que, aunque se valora el trabajo en red y se le asigna importancia a la comunidad y contexto de vida de los adolescentes, cuando se llega a abordar se hace sin mayor formación, asumiendo que este trabajo lo puede realizar cualquier profesional sin mayor preparación para ello. En la mayoría de los programas se observa la ausencia de una estrategia intencionada de mediano y largo plazo, orientada a fortalecer redes y a trabajar con agentes comunitarios. Esto es atribuido a diversos factores, tales como la excesiva carga administrativa, falta de recursos humanos y financieros, el escaso valor asignado a la comunidad y la gestión de las redes a nivel institucional, al minuto de evaluar los indicadores de desempeño en cada programa, etc.

Durante el proceso, en las tres regiones se destaca en los programas una creciente incorporación de la dimensión relacional y contextual, a partir de diversas estrategias para su abordaje planificado. Del mismo modo, se constata la inclusión de estas dimensiones en las planificaciones anuales de varios de los equipos involucrados, quienes avanzan en delimitar roles y funciones asociadas a la realización de tareas de gestión, práctica e intervención en redes.

(...) se considera al joven dentro de un contexto socio comunitario que se constituye en un marco de referencia para el adolescente en su etapa del desarrollo, actuando éste como un factor de riesgo o de protección en los diversos casos. Es por esto que se incorpora en la propuestas de plan de intervención como una área específica, en una primera instancia se centra en el diagnóstico, mediante la construcción histórica del joven relativa a su vinculación a las diversas redes, a nivel de instituciones; nivel escolar, judicial, salud y red SENAME. Como a nivel de redes comunitarias y familiares, identificando la utilización y la falta de las mismas, con el objetivo de poder activarlas en el caso de ser requeridas por los jóvenes. (Equipo de intervención medio libre, Región de la Araucanía)

Hacia el final del estudio, los equipos involucrados refieren que para el abordaje de los aspectos contextuales en la intervención con adolescentes infractores de ley, se requiere de profesionales especializados que cuenten con un repertorio amplio de metodologías, estrategias y herramientas 
organizado en un modelo de trabajo, y que a su vez se integre de modo coherente al modelo general de intervención definido por un equipo. Algunos equipos asumen el desafío de ir especializando el trabajo en esta área.

Hay avance en T2 en la construcción de relaciones de mayor coordinación al interior de los programas, en la medida en que se comparte un enfoque común de trabajo. Se avanza parcialmente en la complementariedad e intersectorialidad, especialmente a nivel de comunas, integrando instituciones vinculadas al tratamiento de drogas, sector justicia, capacitación, educación, salud, por referir algunas. Hay avances relevantes en la coordinación entre los integrantes de la mesa de trabajo (red de programas que aplican sanciones penales para los adolescentes), para compartir información de recursos o procedimientos. Un número menor de programas realiza acciones en en el espacio local, para integrar diferentes recursos comunitarios.

De un modo global, el balance del proceso muestra que para que los avances alcanzados puedan mantenerse, y eventualmente puedan ser transferidos a otros lugares, se requiere garantizar ciertas condiciones y mecanismos para el funcionamiento de las redes. Entre ellos se pueden referir: condiciones básicas para la operación; planificación compartida entre programas y al interior de los programas en el ámbito de las redes; coordinación con una diversidad de actores sociales relacionados con el proceso de intervención; potenciación y fortalecimiento de las redes a través de procesos de reflexividad, aprendizaje continuo y generación y utilización estratégica de conocimiento contextual.

\section{Estrategia construida durante el proceso para mejorar la gestión de redes}

Cabe destacar que en cada una de las regiones se configura en distintos momentos del proceso lo que se denomina "mesas de trabajo", formadas por la red de programas que aplican las sanciones y representantes de la Unidad de Justicia Juvenil de cada una de las direcciones regionales del SENAME. Esta estructura opera en los casos de dos regiones como un grupo motor que planifica, evalúa, monitorea acciones y distribuye responsabilidades. Constituye una red asociativa que permite definir estratégicamente prioridades y actividades, genera cursos de acción de mayor nivel de creatividad, pone a disposición de la mesa el propio capital social de los programas y profesionales para avanzar en los objetivos compartidos.

Cada una de estas mesas resuelve una estructura operativa que permite constituir un frente común para abordar problemas compartidos. Esto lo realiza generando un proceso constante de reflexión y acción. El trabajo desarrollado por esta estructura organizativa logra complementar las funciones de la Unidad de Justicia Juvenil de cada una de las regiones (que tiene como una de sus funciones la de coordinación intersectorial), realizando sucesivas "conversaciones" con encargados de otras líneas institucionales para requerir información sobre sus prestaciones a la población atendida por los programas de la Ley de Responsabilidad Penal Adolescente, sensibilizando sobre las necesidades específicas de esta población y acordando mecanismos específicos para resolver dificultades u optimizar la coordinación y calidad del servicio o recursos brindados a los adolescentes. Se va generando en simultáneo una racionalización de las acciones a nivel local, provincial y regional, distinguiéndose roles y funciones entre las y los involucrados.

Mejores avances fueron obtenidos en aquellos lugares donde los equipos de diferentes programas ya tenían una cierta trayectoria de trabajo compartido, con conocimiento mutuo, mayor apertura al aprendizaje y con encargados de la Unidad de Justicia Juvenil más involucrados y en sintonía con el trabajo compartido con los equipos.

\section{Cambios estructurales en la constitución de las redes sociales}

Dadas las características particulares de cada región, se observan diversos cambios a nivel estructural en las redes interinstitucionales, a partir del proceso de intervención. En específico, la región de Los Ríos es la zona en la que más cambios a nivel estructural se registran, con un progresivo aumento de los nodos de la red, a partir de la diversificación 


\section{Redes T1 PLAE Ranco}
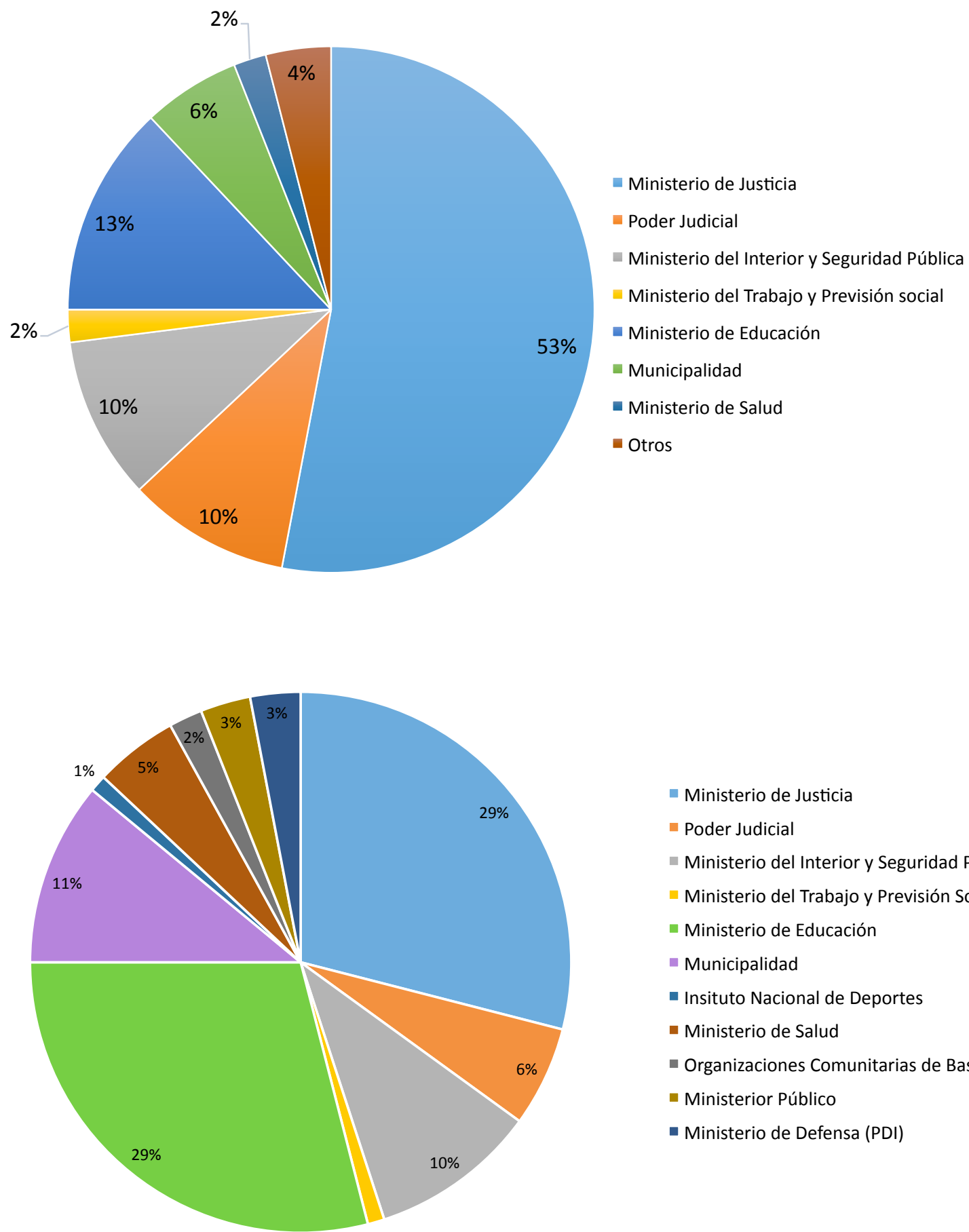

Ministerio de Justicia

- Poder Judicial

Ministerio del Interior y Seguridad Pública

Ministerio del Trabajo y Previsión Social

Ministerio de Educación

- Municipalidad

- Insituto Nacional de Deportes

- Ministerio de Salud

- Organizaciones Comunitarias de Base

- Ministerior Público

- Ministerio de Defensa (PDI)

Figura 2. Incremento y diversificación a nivel institucional en T2 vs. T1.

Fuente: elaboración propia 

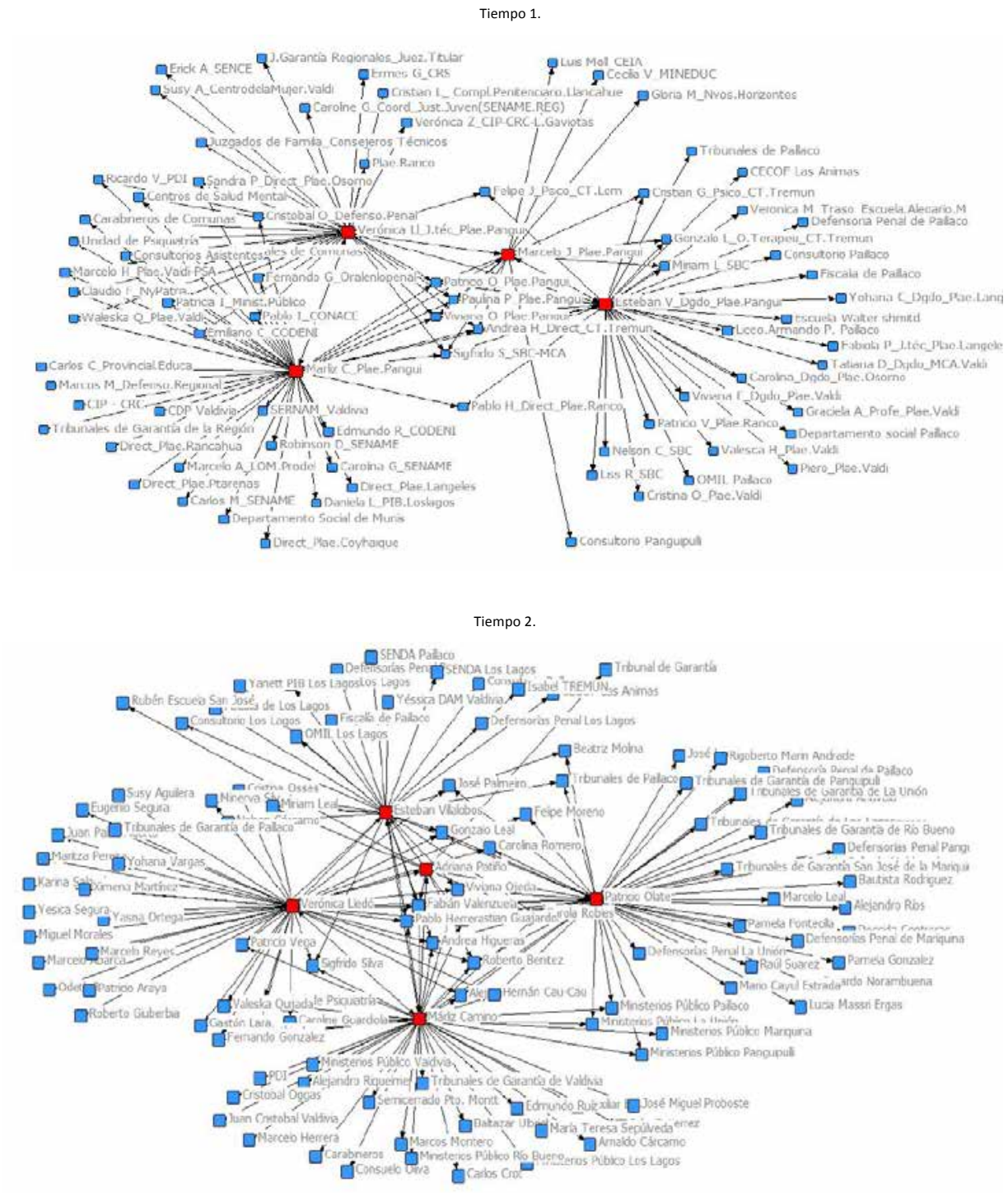

Tiempo 1

Tiempo 2

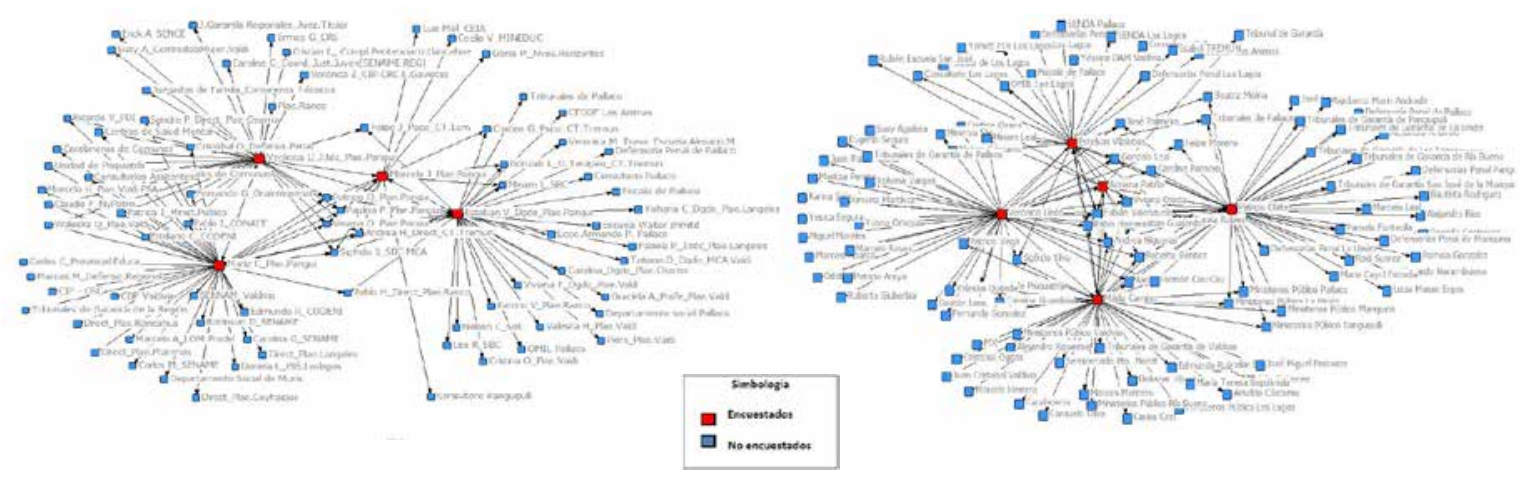

Figura 3. Densificación y aumento de actores del medio [Red un programa de la región de los Ríos].

Fuente: elaboración propia 
de los actores del medio con los cuales los programas se vinculan para resolver los requerimientos de la intervención; esto está además asociado a una progresiva densificación de los vínculos entre los actores. A continuación, se presentan dos gráficos a modo de ejemplo en los cuales se refleja el incremento y diversificación a nivel institucional en el T2 respecto al T1, en el programa "Libertad Asistida del Ranco" de la región de los Ríos (Figuras 2 y 3$)$.

En las tres regiones, se registran cambios en las características de los actores del medio con los cuales se vinculan, identificándose actores con mayores niveles de acceso a toma de decisiones o con cargos directivos dentro de sus instituciones de pertenencia.

\section{Cambios en la dinámica de las redes sociales}

Es posible evidenciar modificaciones en la estructura de algunos equipos que tienen impacto en la dinámica interna y externa de funcionamiento. Algunos equipos resuelven incluir un encargado o gestor de redes (ya sea contratando o reasignado a una persona del equipo para cumplir ese rol). De forma complementaria, se definen flujos de acción y aportes diferenciales de parte del resto del equipo y la dirección en el área, definiendo de forma progresiva una forma de hacer en este ámbito. Una variante ha sido que diferentes integrantes del equipo cumplan funciones en este ámbito, abordando temáticamente esas relaciones. Así, además de gestionar la intervención específica de dicha área (por ejemplo, educación), se ocupan de la gestión de las redes en ese ámbito específico.

En el caso de educación generamos un proceso de intervención específico en esa área con una persona que está encargada del área (...). El gestiona las rede específicas de educación y resulta muy bien, maneja un lenguaje común con los actores de educación, y permite hacer las gestiones mucho más rápido y de forma más operativa. Genera cercanía porque el otro te observa como un par más o menos cercano, no como otro lejano. (Equipo de intervención medio libre, Región de la Araucanía)

Se evidencia, además, un aumento significativo del porcentaje de eficiencia en la vinculación en las redes que establecen los programas y centros con el medio. Esto se puede observar al medir el porcentaje inicial de eficiencia de la red total y compararlo con el porcentaje total de eficiencia de las redes actuales. A continuación un ejemplo con la región de Los Lagos en la Tabla 1 que refiere cambios en la eficiencia de la red.

\section{Mecanismos para el funcionamiento de las redes sociales}

A partir de los resultados obtenidos, es posible concluir que si bien se pueden apreciar avances en la gestión y en el trabajo de redes, para que estos

TABLA 1

Cambios en la eficiencia de la red

\begin{tabular}{lcccccc}
\hline \multicolumn{3}{c}{ Eficiencia de la red antes-después, programas medio libre región de Los Lagos } \\
\hline & \multicolumn{7}{c}{ \% antes } & \multicolumn{4}{c}{ \% después } \\
\hline & Ineficiente & Medianamente eficiente & Eficiente & Ineficiente & Medianamente eficiente & Eficiente \\
\hline Programa 1 & 0 & 16.67 & 83.33 & 0 & 11.9 & 88.1 \\
\hline Programa 2 & 0 & 4.76 & 95.24 & 5.88 & 23.53 & 70.59 \\
\hline Programa 3 & 0 & 18.52 & 81.48 & 0 & 4.44 & 95.56 \\
\hline Programa 4 & 0 & 15.15 & 84.85 & 0 & 5.63 & 94.37 \\
\hline Programa 5 & 0 & 33.33 & 66.67 & 0 & 40.63 & 100 \\
\hline Programa 6 & 0 & 32 & 68 & 0 & 14.36 & 59.38 \\
\hline \% Totales & 0 & 20.07 & 79.93 & 0.98 & 84.66 \\
\hline
\end{tabular}

Fuente: elaboración propia 
avances sean posibles y extensibles, se requiere expresamente de personas que se encarguen de la tarea de abordar las redes de forma sistemática, conocimiento teórico y práctico acerca de cómo realizar dichas acciones, convenios operativos que provean un marco para la acción en términos de recursos disponibles y una organización del equipo de trabajo para el abordaje del contexto relacional.

Los programas deben organizarse entre sí para establecer mecanismos de planificación compartida en el área de redes, debido a que hay casos, recursos y espacios de intervención que les son comunes y que suponen complementariedad y cooperación. Esto supone que los programas planifiquen de forma interna las estrategias de intervención en el ámbito de redes, para que se generen procesos de largo plazo que sean evaluables y que formen parte de las labores cotidianas de los miembros del equipo. Así mismo, se requiere generar estrategias de coordinación con el entorno y estrategias continuas de reflexión al interior de los equipos y entre ellos, de modo de sostener un aprendizaje continuo que permita la retroalimentación, asegurando la mejora permanente de las prácticas.

Es una constatación también, que deben existir indicaciones claras desde la institucionalidad pública correspondiente (en este caso SENAME) acerca de la importancia de la incorporación de las dimensiones del contexto, pero ello además debe acompañarse de indicadores que valoren efectivamente el trabajo en esta línea.

\section{Discusión}

A partir de los resultados del estudio, es posible sostener que hay avances en la operatividad de las redes de colaboración en distintos niveles (regional y local), con diferentes alcances y proyecciones según región y tipos de programas, evidenciándose notorias diferencias en los avances entre el medio privativo de libertad respecto de las modalidades de sanción en medio libre. Se entiende que estos avances se inscriben en un proceso complejo que requiere de acciones concretas de consolidación con proyección a largo plazo, por lo que exige mayor tiempo para dimensionar su real alcance. Cabe destacar también la presencia previa en algunas regiones de un importante nivel de capital humano y capital social que venía sosteniendo redes de colaboración más estables, por lo que el proceso permitió extender la especialización a otros equipos, sostener un trabajo más sistemático y coordinado y especialmente que la Unidad de Justicia Juvenil de SENAME asumiera un rol más protagónico en el liderazgo técnico del proceso en una lógica de colaboración.

Pero según se puede apreciar en el estudio, atender a lo contextual en cada programa depende de un conjunto de condiciones. De un lado, que estén resueltas urgencias en lo relativo a la gestión interna del equipo: constitución, liderazgo técnico claro y recursos básicos para operar. A ello se suma el valor de la experiencia del equipo en el territorio y el nivel de conocimiento de los recursos disponibles en él. Se requiere, además, de cierto nivel de formación que faculte al equipo compartir un marco comprensivo común y estrategias técnicas en el plano práctico y operativo que permitan visualizar de forma conjunta el fenómeno de las redes y actuar en ellas de forma efectiva. En la investigación, aparece como necesidad que la Unidad de Justicia Juvenil del SENAME apoye el proceso de concertación de redes institucionales en cada región, lo que supone estabilidad laboral y la especialización de las o los profesionales que la integran.

Es una constatación también, que deben existir indicaciones claras desde la institucionalidad pública correspondiente (en este caso SENAME), acerca de la importancia de la incorporación de las dimensiones del contexto, pero ello además debe acompañarse de indicadores que valoren efectivamente el trabajo en esta línea.

Cabe anotar que esta experiencia, que muestra avances particularmente al interior de los equipos en algunas redes regionales y locales, presenta el desafío de seguir sosteniendo un trabajo intencionado, con capacidad de plantearse una visión compartida y trazar objetivos a corto y largo plazo, con un liderazgo claro (no necesariamente de personas particulares).

Los resultados de la investigación permiten resaltar la relevancia de la colaboración entre los 
diferentes actores del sistema que enfrentan los desafíos de la política pública en este ámbito específico (Luna \& Velasco, 2005; Uvalle, 2009), que la calidad y densidad de las relaciones que la hacen posible pueden y deben ser favorecidos de modo rigurosos y participativo, en un proceso que, como deja de relieve esta investigación, no se improvisa. Trabajar de modo coordinado mejorando la continuidad y complementariedad de la intervención con los adolescentes infractores de ley, favorece el uso sinérgico y estratégico de recursos que normalmente no están disponibles para los jóvenes y sus familias (Pérez-Luco et al., 2014; Zambrano, Muñoz et al., 2014), pero ello supone también especialización e intercambio en el sistema.

Generar un soporte contextual para reducir el riesgo de reincidencia criminógena, pero también y fundamentalmente para generar condiciones de inclusión social para estos jóvenes, supone integrar de modo coherente ámbitos fundamentales para la vida de este grupo, como lo son la familia, el barrio y las redes focales que lo vinculan (Dionne $\& \mathrm{Al}$ tamirano, 2012).

La adhesión de las y los profesionales a las nuevas prácticas que deben ser implementada necesita de un tiempo más o menos largo y está fuertemente determinada por las estrategias de difusión (informar y persuadir) puestas en marcha respecto de esas nuevas prácticas (LeBlanc \& Robert, 2012). En coherencia con esto, la metodología de investigaciónacción es apropiada, en la medida que incorpora un fuerte componente participativo, de formación y reflexión que permite un ir y venir constante entre la práctica y la teoría, con la finalidad de innovar y mejorar de acuerdo a las realidades de cada equipo, programa e institución en particular.

Un aspecto crítico que transversalmente afectó la sistematicidad del trabajo fue el cambio reiterado de autoridades en cada una de las regiones, así como de encargados e integrantes de la Unidad de Justicia Juvenil, lo que fue limitando las posibilidades de avanzar con coherencia, pues cada autoridad y profesional tenía niveles de conocimiento, compromiso y apertura diferente ante el tema y el proyecto en particular. En esta línea, es necesario contar con sistematización de las prácticas, así co- mo con un modelo general de abordaje que pueda ser transferido de forma sistemática a los nuevos profesionales y directivos de esta política social, de modo de facilitar una mejor incorporación de las personas que comúnmente rotan en este ámbito laboral, disminuyendo la variabilidad en los procesos de inducción y facilitando un accionar coherente e integrador.

Finalmente, destacar que el tipo de metodología empleada en el proceso llevado a cabo en conjunto con los organismos involucrados en la experiencia permite valorar el rigor de los procesos investigativos, así como lo complejo e impredecible del trabajo en terreno (Dionne \& Altamirano, 2012), cuestiones fundamentales para documentar y generalizar las buenas prácticas. En este caso, la experiencia ha permitido generar un manual de Intervención, Práctica y Gestión de Redes (Zambrano, Muñoz et al., 2014) que apunta a compartir un modelo operativo adaptable a las realidades particulares de los programas, redes interinstitucionales y particularidades de los territorios en donde se lleva a cabo el trabajo con los jóvenes.

Un desafío investigativo ulterior sería efectuar un seguimiento a los equipos en las tres regiones para observar el grado de sustentabilidad de los avances pesquisados en esta investigación, considerando la rotación del recurso humano que se evidencia en los programas, dado que el financiamiento se encuentra supeditado a procesos de licitación que restan estabilidad a los equipos. Así también, replicar experiencias similares empleando el modelo operativo generado, atendiendo a los procesos involucrados en la innovación. Un desafío adicional sería avanzar de la integración de las redes institucionales (ámbito en que se aprecian mayores logros en este estudio) hacia la integración de las redes comunitarias y focales, dimensiones en las que se aprecian mayores dificultades en la investigación.

\section{Referencias}

Bonet i Martí, J. (2006). La vulnerabilidad relacional: análisis del fenómeno y pautas de intervención. REDES-Revista Hispana para el Análisis de Redes Sociales, 11(4), 1-17. 
Canales, M., Fuentealba, T., Jiménez, J., Morales, J., Cottet, P., \& Agurto., I. (2005). Factores que inciden en la comisión de delitos graves en adolescentes infractores de ley. Revista El Observador, 1, 49-72.

Contreras, J., Rojas, V., \& Contreras, L. (2015). Análisis de programas relacionados con la intervención en niños, niñas y adolescentes vulnerados en sus derechos: la realidad chilena. Psicoperspectivas, 38(2), 327-342.

Claes, M., Lacourse, E., Ercolani, A. P., Pierro, A., Leone, L., \& Presaghi, F. (2005). Parenting, peer orientation, drug use, and antisocial behavior in late adolescence: A cross-national study. Journal of Youth and Adolescence, 34(5), 401-411.

Crespi, M., \& Mikulic, I. (2009). Reinserción social de liberados condicionales: análisis de la dimensión relacional desde el enfoque de redes de apoyo social. Anuario de Investigaciones, 16, 211-221.

Del Piano, C. (2011). Desafíos para la coordinación Interinstitucional del ministerio del interior y Seguridad Pública. Tesis para la obtención del grado de Magíster en Gestión y Políticas Públicas, Ingeneria Industrial, Universidad de Chile.

Dionne, J., \& Altamirano, C. (2012). Los desafíos de un verdadero sistema de justicia juvenil: una visión psicoeducativa. Universitas. Psychologica, 11(4), 1055-1064.

Dionne, J., \& Zambrano, A. (2009). Intervención con adolescentes infractores de ley. Revista El Observador, 5, 35-56.

Fleury, S. (2002). El desafío de la gestión de las redes de políticas. Revista Instituciones y Desarrollo, 12-13, 221-247. Disponible en http://www. academia.edu/ 4350411/FLEURY_S._El_desafio_de_la_gesti\%C3\%B3n_de_las_redes_de_ pol\%C3\%ADticas

Fréchette, M., \& Le Blanc, M. (1998). Délinquances et délinquants (8.. ed.). Québec: Gaëtan Morin.

Gimeno, R., \& Nogueras, A. (2013). Programa de suport a la prevenció de la delinqüencia juvenil i a la mediació comunitaria. Documento de trabajo, Servei de Mediació i Assessorament Tècnic Generalitat de Catalunya, Departament de Justícia Direcció General d'Execució Penal a la Comunitat i Justícia Juvenil. Disponible en http://justicia.gencat.cat/ web/.content/home/ambits/formacio__ recerca_i_ docum/formacio/gestcon/comunitats_de_practica/ prevencio_i_mediacio/program_suport_prev_i_ med2011.pdf

Gendreau, P., Goggin, C., Cullen, F. T., \& Andrews, D. A. (2000). The effects of community sanctions and incarceration on recidivism. Forum on Corrections Research, 12(3), 10-13.

Gracia, E., \& Herrero, J. (2006). La comunidad como fuente de apoyo social: evaluación e implicaciones en los ámbitos individual y comunitario. Revista Latinoamericana de Psicología, 38(2), 327-342.

Hein, A. (2002). Factores de riesgo y deincuencia juvenil: revisión de la literatura nacional e internacional. Santiago de Chile: Paz Ciudadana.

Hoge, R. D., \& Andrews, D. A. (1994). The Youth Level of Service/Case Management Inventory and manual. Ontario: Carleton University.

Holt, T., Bossler, A., \& May, D. (2011). Low self-control, deviant peer associations, and juvenile cyberdeviance. Southern Criminal Justice Association, 37(3), 378-395.

LeBlanc, L., \& Robert, M. (2012). La innovación psicosocial: planificar su implementación y difusión para prevenir la delincuencia juvenil. Universitas Psychologica, 11(4), 1125-1134.

LeBlanc, L., Swisher , R., Vitaro, F., \& Tremblay, R. (2007). School social climate and teachers perceptions of classroom behavior problems: A 10 year longitudinal and multilevel study. Social Psychology of Education, 10(4), 429-442.

LeBlanc, M. (2006). Self-control and social control of deviant behavior in context: Development and interactions along the lief course (A. Emaldía, Trad.). En P. -O. Wikström \& R. Sampson (Eds.), The esplanation of crime: Context, mechanisms, and development (pp. 195-242). Cambridge: Cambridge University Press.

Luna, M., \& Velasco, J. (2005). Confianza y desempeño en las redes sociales. Revista Mexicana de Sociología, 67(1), 127-162.

Martínez, J. (2010). Informe de diagnóstico de la implementación de la Ley 20.084 Junio 2007-Marzo 2010. Santiago: SENAME.

Martínez, V. (2006). El enfoque comunitario. El desafío de incorporar a la comunidad en las intervenciones sociales. Santiago: Universidad de Chile. 
EL DESAFÍO DE INCORPORAR LAS REDES INSTITUCIONALES Y COMUNITARIAS EN LA INTERVENCIÓN CON ADOLESCENTES INFRACTORES: UNA INVESTIGACIÓN-ACCIÓN EN TRES REGIONES DEL SUR DE CHILE

Méndez, P., \& Barra, E. (2008). Apoyo social percibido en adolescentes infractores de ley y no infractores. Psykhe, 17(1), 59-64.

Montero, M. (2005). Introducción a la psicología comunitaria. Desarrollo, conceptos y procesos. Buenos Aires: Paidós.

Moreira, V., Sánchez, A., \& Mirón, L. (2010). El grupo de amigos en la adolescencia. Relación entre afecto, conflicto y conducta desviada. Boletín de Psicología, 100, 7-21.

Pérez-Luco, R., Alarcón, P., Zambrano, A., Alarcón, M., Lagos, L., Wenger, L., ... Reyes, A. (2014). Manual de intervención diferenciada. Prácticas que transforman vidas. Temuco: Universidad de La Frontera.

Repetto, F. (2010). Coordinación de políticas sociales: abordaje conceptual y revisión de experiencias latinoamericanas. Centro Latinoamericano de Administración para el Desarrollo.

Rendón, E. (abril, 2013). La prevención de la delincuencia juvenil en el Programa Todos somos Juárez: hacia una nueva estrategia de seguridad y prevención de violencias en México. Ponencia presentada en Foro Internacional de Buenas Prácticas en Prevención de la Delincuencia Juvenil, Bogotá.

Rezende, M., \& Estevão, R. (2012). Juvenile criminal behavior and peers' influences: A comparative study in the Brazilian context. Universitas Psychologica, 11(4), 1157-1166.

Rodríguez, J. (2011). Conducta antisocial en grupo: una aproximación mediante ecuaciones estructurales. Revista Cenipec, 30, 237-264.

Rodríguez, J., \& Mirón, L. (2008). Grupo de amigos y conducta antisocial. Capítulo Criminológico, 36(4), 121-149.

Sandoval, C. (2007). Relatos de vida de jóvenes infractores de ley: una aproximación a sus procesos de reinserción social y comunitaria (Tesis de Magíster no publicada). Universidad de Chile, Santiago de Chile.

Serrano, S., Azocar, B., Dorvillius, E., Echevarria, P., Filliaudeau, H., Jamoulle, P., \& Joubert, M. (2009). Elementos generales para entender la generación de conductas de riesgo en sectores vulnerables
(S. Serrano, Trad.) [Edición especial]. Revista El Observador, 5, 120-167. Disponible en http://www. sename.cl/wsename/otros/observador5/el_observador_5_119-168.pdf

Uvalle, R. (2009). Gestión de redes institucionales [Numero especial]. Convergencia,16, 41-72.

Valdenegro, Boris Andrei. (2005). Factores Psicosociales Asociados a la Delincuencia Juvenil. Psykhe (Santiago), 14(2), 33-42. Recuperado en 05 de febrero de 2015, de http://www.scielo.cl/scielo.php?script=sci_ arttext\&pid=S0718-22282005000200003\&lng =e s\&tlng=es. 10.4067/S0718-22282005000200003.

Vega, M. J. (2012). El programa ambulatorio de discapacidad del Servicio Nacional de Menores, desde la mirada de un modelo de análisis de política pública (Tesis de Magíster no publicada). Universidad de Chile, Santiago de Chile.

Veillet, N., \& Laporte, F. (2010). Continuidad de los servicios. Presentación efectuada en el marco del Seminario Internacional de Intervención con Infractores de la Ley, Ribeirão Preto, São Paulo, Brasil.

Vieira, P. (abril, 2013). Programa Escohas: inclusión social de niños y jóvenes en Portugal: las contribuciones en 12 años de intervención. Ponencia presentada en Foro Internacional de Buenas Prácticas en Prevención de la Delincuencia Juvenil, Bogotá.

Zambrano, A., Betancourt, M., Jerez, P., Ortiz, C., \& Soto, C. (2014). Componentes y criterios fundamentales para estrategias de prevención comunitaria de desadaptación social y comportamiento de riesgo adolescente: perspectiva de expertos internacionales. Revista Señales, 8(13), 6-30.

Zambrano, A., Muñoz, J., \& Andrade, C. (2014). Manual de intervención, práctica y gestión en redes. Tejiendo oportunidades. Temuco: Universidad de La Frontera.

Zambrano, A., Muñoz, J., \& González, M. (2012). Variables psicosociales del entorno comunitario asociadas a procesos de desadaptación social en adolescentes: reflexiones a partir de un estudio de caso. Universitas Psychologica, 11(4), 1135-1145. 
